\title{
Educação sexual no ensino básico: o estudante de medicina como educador
}

\author{
Sex education in middle schools: the medical student as an educator \\ La educación sexual en la educación básica: el estudiante de medicina como educador \\ Clarissa Garcia Custódio", Felipe Leonardo¹, Helena Boldrini Niero¹, Luisa Hercowitz Tagnin¹, Luiza \\ Lista Bertonha ${ }^{1}$, Rebeca da Silva Sousa ${ }^{1}$, Eunice Maria de Toledo Damito ${ }^{1}$, Maria Valéria Pavan ${ }^{1 *}$.
}

\section{RESUMO}

Objetivo: Descrever a experiência com um projeto que discute desenvolvimento sexual com alunos do Ensino Básico da rede pública, conduzido por estudantes de medicina. Relato de Experiência: Em prática desde 2016, o projeto vem sendo organizado em oficinas desenvolvidas com alunos e seus pais ou responsáveis, capacitação de professores e fornecimento de material para que possa ser replicado pelas escolas. Em 2020, passou por adaptações para ser mediado por plataformas digitais. Em conjunto com os professores, foi produzido um vídeo sobre o desenvolvimento do corpo humano, com foco nas alterações físicas da puberdade; uma aula sobre o comportamento durante o desenvolvimento, mostrando como as crianças respondem às diferentes fases do crescimento e uma história em quadrinhos, online, sobre uma adolescente com suspeita de gravidez que decide ir pela primeira vez a uma consulta na Unidade Básica de Saúde. Considerações Finais: Diferentemente da abordagem preventiva, heteronormativa, graduada de acordo com a idade dos alunos e usualmente inserida como um tema das aulas de ciências, esse projeto procura dar aos professores, família e sistema de saúde local a oportunidade de se apropriarem da tarefa da discussão sobre educação sexual, com reconhecimento dos obstáculos, dos preconceitos e ganho de autonomia.

Palavras-chave: Educação em saúde, Educação sexual, Adolescentes.

\begin{abstract}
Objective: To describe the experience with a project that discusses sexual development with middle school students from public schools, conducted by medical students. Experience Report: In practice since 2016, the project has been organized in workshops developed with students and their parents or guardians, teacher training and provision of material so that it can be replicated by schools. In 2020, has undergone adaptations to be mediated by digital platforms. Together with the teachers, a video on the development of the human body was produced, focusing on the physical changes of puberty; a virtual presentation on behavior during development, showing how children respond to different stages of growth, and an online comic about a teenager with a suspected pregnancy who decides to go to the Primary Health Care Unit for the first time. Final Considerations: Unlike the preventive, heteronormative approach, graduated according to the age of the students and usually inserted as the theme of science classes, this project seeks to give teachers, family and the local health system the opportunity to appropriate the task of discussing education with the recognition of obstacles, prejudices and gaining autonomy.
\end{abstract}

Keywords: Health education, Sex education, Adolescent.

\section{RESUMEN}

Objetivo: Describir la experiencia con un proyecto que discute el desarrollo sexual con estudiantes de primaria de escuelas públicas, realizado por estudiantes de medicina. Informe de Experiencia: En la práctica desde 2016, el proyecto se ha organizado en talleres desarrollados con los alumnos y sus padres, la formación del profesorado y la provisión de material para que pueda ser replicado por las escuelas. En 2020, sufrió adaptaciones para ser mediado por plataformas digitales. Junto con los profesores, se produjo un video sobre el desarrollo del cuerpo humano, centrado en los cambios físicos de la pubertad; una clase sobre comportamiento durante el desarrollo, donde se muestra cómo responden los niños a las diferentes etapas

${ }^{1}$ Pontifícia Universidade Católica de São Paulo (PUC/SP), Sorocaba - SP. *E-mail: mvpavan@globo.com 
del crecimiento, una historieta, en línea, sobre una adolescente con sospecha de embarazo que decide acudir a una primera visita a la Unidad Básica de Salud. Consideraciones Finales: A diferencia del enfoque preventivo, heteronormativo, graduado de acuerdo a la edad de los estudiantes y generalmente insertado como tema de las clases de ciencias, este proyecto busca brindar a los docentes, la familia y el sistema de salud local la oportunidad de apropiarse de la tarea de discutir la educación con reconocimiento de obstáculos, prejuicios y ganando autonomía.

Palabras clave: Educación en salud, Educación sexual, Estudiantes.

\section{INTRODUÇÃO}

As Diretrizes Curriculares Nacionais do Curso de Graduação em Medicina (DCN) preconizam a inserção do estudante de medicina no Sistema Único de Saúde (SUS) logo nos primeiros semestres do curso (BRASIL, 2014). Atendendo a essas orientações, os alunos de um curso de medicina do interior de São Paulo são inseridos nas atividades de Atenção Primária à Saúde (APS) desde o primeiro ano do curso. Com isso, eles têm a possibilidade de participar da discussão e busca de soluções para os problemas identificados na área em que estão atuando, através da observação da realidade (POLES TPG, et al., 2018). A saúde sexual dos adolescentes é um grande problema de saúde pública e encontra no Brasil diversas barreiras para seu enfrentamento (GAVA T e VILLELA WV, 2016; FURLANETTO MF, et al., 2018).

Diante disso, desde 2016 alunos do curso de medicina vêm trabalhando junto à comunidade e, a partir da demanda apontada pelos adolescentes, equipes de saúde e escolas, buscam compreender os fatores de risco para gravidez na adolescência e propor, em parceria com as escolas, ações que possam contribuir para o acesso à informação segura sobre desenvolvimento sexual e para mudanças no modelo de educação em saúde sexual, que na maioria das vezes se organiza numa prática burocrática e pouco reflexiva (MARCON $\mathrm{N}$, et al., 2020; FURLANI J, 2011). A necessidade de introduzir a educação sexual no ensino formal de crianças e adolescentes já vem sendo discutida e implementada desde o início do século passado, quando, entretanto, era feita sob a ótica sanitarista e de uma forma autoritária (FURLANETTO MF, et al., 2018).

Após décadas de discussões e mudanças, os Parâmetros Curriculares Nacionais (PCN), instrumento do Ministério da Educação que normatiza os conteúdos ensinados na educação básica, colocaram a educação sexual como um tema transversal, que deve ser integrado às diversas disciplinas e abordado por meio de problematização e diálogo, de maneira que a escola possa exercer sua função no máximo de suas potencialidades, incluindo sua função social (BRASIL, 1998). Entretanto, essa tem sido uma meta difícil de ser alcançada (FURLANETTO MF, et al., 2018).

Diante do exposto, o objetivo desse trabalho foi descrever a experiência com um projeto que discute desenvolvimento sexual com alunos do Ensino Básico da rede pública, conduzido por estudantes de medicina.

\section{RELATO DE EXPERIÊNCIA}

Trata-se do relato de experiência de um projeto que utiliza metodologias ativas para discutir desenvolvimento sexual com alunos do Ensino Básico de escolas públicas de um município de grande porte no interior do Estado de São Paulo. Em prática desde 2016, este projeto vem sendo ampliado ao longo dos anos e, em 2020, foi estendido para atuar em quatro escolas do município, contemplando 1000 crianças e adolescentes, além de atividades com os pais e responsáveis pelos alunos. Projeto aprovado pelo Comitê de Ética em Pesquisa (CAAE: 30531620.8.0000.5373). O grupo de trabalho em 2020 conta com a participação de uma aluna e um aluno do quarto ano de medicina, que coordenam os trabalhos; quatro alunas de iniciação científica, do segundo e terceiro anos do curso de medicina e duas professoras coordenadoras.

A escolha desses alunos é realizada por processo de seleção como forma de garantir o interesse, disposição e compromisso com o projeto, que segue um planejamento semanal com reuniões, discussões temáticas, escrita de documentos para cumprimento dos editais e execução. O projeto é aberto para outros alunos de graduação que estejam em atividade na área e tenham interesse em participar, como parte do projeto de intervenção previsto na matriz curricular. $O$ projeto utiliza oficinas que são organizadas com as 
seguintes atividades: A) aplicação de um questionário, sem identificação do respondedor, e rodas de conversa para conhecer os alunos participantes, o nível de informações prévias e o desejo de informações sobre o desenvolvimento sexual.

A coleta desses dados contribuem para a organização das demais atividades; B) uma peça de teatro interativa, para cativar os estudantes presentes e discutir questões sobre a gravidez na adolescência, a importância dos cuidados médicos, o papel da Unidade Básica de Saúde (UBS) nesse contexto, as relações de cuidado, preconceitos e empoderamento feminino frente a essa situação; C) uma atividade utilizando a planilha dos quatro campos da vida (social; escola e trabalho; saúde física e mental; finanças e dinheiro), onde os alunos podem discutir em pequenos grupos os impactos positivos e negativos decorrentes de uma gravidez na adolescência, suas experiências diante da realidade e posteriormente discutir com toda a sala; D) uma dinâmica para que os adolescentes, por meio de escolha aleatória, possam entender e explicar o uso de métodos contraceptivos que ficam disponíveis durante a oficina, tais como preservativo masculino, preservativo feminino, pílula anticoncepcional, assim como absorventes femininos; E) um jogo de perguntas e respostas em equipes sobre puberdade, sexualidade, gênero, saúde pública e direitos dos adolescentes; F) apresentação de peças anatômicas de pelve feminina e masculina.

As oficinas são programadas para serem realizadas em 40 minutos, para que ocupem o período de uma aula, evitando comprometimento da agenda da escola e para que possam ser utilizadas pelos professores em horário regular de aula. Com o fechamento dos serviços, das escolas e das universidades em função da pandemia da COVID-19, deu-se início às atividades remotas na maioria das instituições de ensino (BRASIL, 2020). Diante disso, os alunos do curso de medicina que estavam ativos nos diversos campos de prática, trabalhando junto à comunidade nas equipes de saúde da família, também tiveram suas atividades bruscamente interrompidas.

Entre elas, as atividades planejadas para serem realizadas nas escolas. Com a retomada das atividades escolares a distância no estado de São Paulo, optou-se por retomar com o projeto. Para isso, foram feitas adaptações, com produção de materiais que pudessem ser veiculadas através dos canais de comunicação em prática no Ensino Básico no estado de São Paulo (SÃO PAULO, 2020). Com o apoio dos professores das escolas foi construído um material que pudesse contribuir com as atividades curriculares, com inserção do tema desenvolvimento sexual, para ser veiculado pelas plataformas digitais, entre elas Facebook, Google Classroom e WhatsApp. O material foi disponibilizado aos alunos a critério dos professores e mediadores das escolas.

Motivados pela pesquisa bibliográfica prévia, orientações da escola e demanda de introduzir a temática, a primeira atividade, sobre o desenvolvimento do corpo humano, foi confeccionada no formato de vídeo, utilizando a ferramenta Sp Adobe Spark. O objetivo foi elucidar os marcos desde o momento da fecundação até a adolescência, abordando anatomia dos órgãos reprodutores, fisiologia da produção dos gametas, de maneira ilustrativa e simplificada. O material tem duração de 5 minutos e 34 segundos, priorizando o uso de imagens para que o conteúdo possa manter a atenção das crianças e adolescentes, sendo de fácil interpretação.

Embora essa atividade se organize com informações bem descritivas do desenvolvimento do corpo humano, houve impedimento pela escola de sua veiculação às crianças de 10 a 13 anos, antecipando a censura dos pais e responsáveis. O material foi utilizado apenas para as aulas para os alunos do nono ano do ensino fundamental e primeiro ano do ensino médio. A segunda atividade, movida pela necessidade de uma nova abordagem, contou com a ferramenta do Microsoft Power Point para composição de uma apresentação em slides que trouxe a teoria do desenvolvimento psicológico e emocional do adolescente, também de forma bastante ilustrativa. Essa aula foi elaborada com o propósito de evidenciar a relação entre as mudanças psíquicas e o desenvolvimento sexual que muitas vezes é negligenciada.

Nessa história, sabendo que o isolamento social vivido devido à pandemia tem levado os adolescentes a ficarem mais tempo em casa e, consequentemente, compartilhar um tempo ainda maior com irmãos mais novos e, frequentemente, ficar responsáveis por eles, principalmente em função do trabalho da mãe, resolveuse abordar também, de maneira lúdica, como lidar com as birras. 
Esse material foi disponibilizado para todos os alunos via Facebook, sem qualquer impedimento em relação à idade. O material também foi utilizado pelos professores como apoio para as atividades regulares. Também foi feito um questionário, criado a partir da plataforma Survey Monkey, para auxiliar o professor no processo de avaliação formativa.

O questionário se organiza em dez perguntas de múltipla escolha ou verdadeiro/falso, com o objetivo de compreender o potencial educativo das atividades desenvolvidas e avaliar o seu impacto no conhecimento dos alunos, e na última sessão traz as respostas corretas, permitindo ao próprio aluno confirmar seus erros e acertos e voltar para a questão.

Por fim, conservando a dinâmica em busca de ferramentas diferentes para a produção do material, foi elaborada uma história em quadrinhos digital sobre uma adolescente com suspeita de gravidez que vai a uma consulta na UBS. Essa atividade foi uma adaptação do roteiro do teatro usado nas oficinas presenciais. $O$ material foi produzido na plataforma Pixton, que possibilita a criação e disponibilização de conteúdo digital de forma fácil.

No contexto dessa atividade, viu-se uma oportunidade de levar aos adolescentes a informação sobre o direito ao atendimento nas UBS, mesmo sem a presença dos pais e responsáveis, indicando, dessa maneira, a possibilidade de atendimento em situações de risco (Figura 1). 
Figura 1 - História em Quadrinhos Digital.

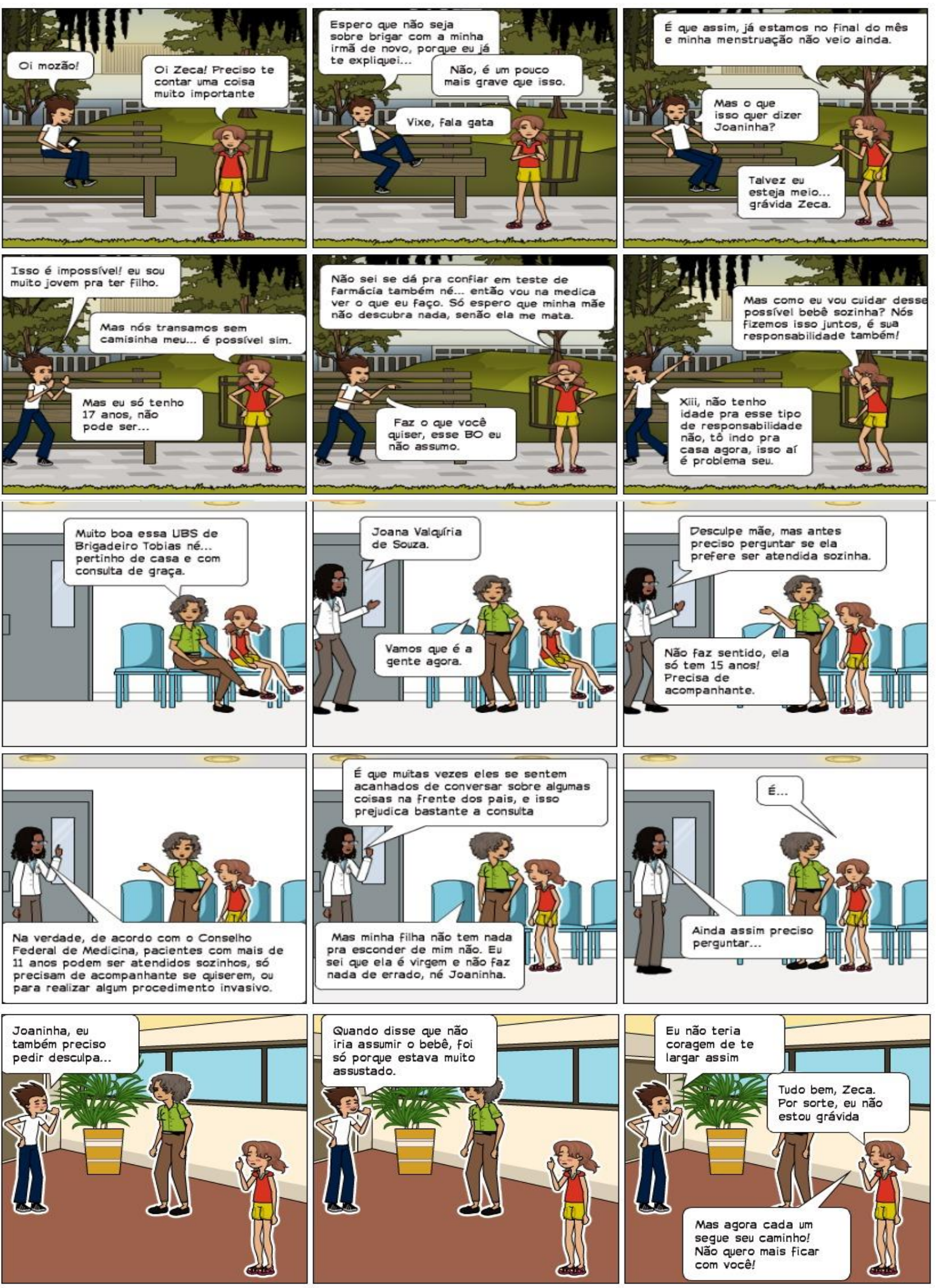

Fonte: Custódio CG, et al., 2020. 


\section{DISCUSSÃO}

A inserção na comunidade, motivada pela mudança no paradigma da educação médica, possibilitou aos acadêmicos e preceptores o reconhecimento do território e das suas potencialidades como campo de prática dialógica, em que as necessidades são discutidas com a comunidade, as propostas de soluções surgem desse diálogo e a universidade se coloca como mais um personagem desse processo (BRASIL, 2014; CESÁRIO RR, et al., 2019).

Neste contexto, a educação sexual aparece como uma demanda dos adolescentes, da equipe de saúde e das escolas, que apontam as dificuldades enfrentadas para a discussão do tema. Esse projeto, em toda sua formatação, foi pensado para ser um instrumento de apoio a essa demanda da comunidade, provendo conteúdo e método para um trabalho conjunto com os professores para abordar o tema desenvolvimento sexual em sala de aula e fora dela.

Em sua revisão sobre a abordagem da sexualidade nas escolas, Furlanetto MF, et al. (2018) mostra que a grande maioria das atividades para discussão do tema são realizadas de forma temporária, ou até mesmo pontual, em grande parte feita por graduandos das áreas da saúde, como projetos de pesquisa ou intervenção, sem articulação com o currículo transversal das escolas. Diferentemente, esse projeto tem como objetivo que os professores e a família, com o auxílio do sistema de saúde local, se apropriem da tarefa da discussão sobre educação sexual, com reconhecimento dos obstáculos e preconceitos impostos pelo meio, com ganho de autonomia.

Assim como discutido por Gesser M, et al. (2015), as atividades sobre saúde sexual nas escolas acontecem com uma abordagem preventiva, heteronormativa e graduada de acordo com a idade dos alunos e é, usualmente, inserida como um tema das aulas de ciências. Entendendo que é preciso conhecer a realidade, para que se possa oferecer propostas de soluções, este grupo busca trabalhar em parceria com a escola e criar espaços para mudanças da prática a partir de oportunidades geradas pelo cotidiano. Com isso, mesmo enfrentando dificuldades, foi possível manter a discussão sobre saúde sexual com os alunos durante a pandemia.

Vários estudos mostram a falta de capacitação dos professores como um fator que pode dificultar a abordagem do tema saúde sexual nas escolas (GAVA T e VILLELA WV, 2016; MARCON N, et al., 2020 GESSER M, et al., 2015; FURLANETTO MF, et al., 2018; FURLANETTO MF, et al., 2019). Neste sentido, as primeiras atividades para a distribuição virtual e remota foram construídas de forma mais conteudista, com a intenção de ser substrato técnico-científico para alunos, mas também para professores.

As demais atividades apontam para a compreensão da sexualidade como uma realidade que transcende os aspectos biológicos e encontra intersecção com a subjetividade pessoal e as relações familiares, com o objetivo de conceder subsídios para um discurso que não se baseie em vieses moralistas. Vieses esses que encontram suporte na crença por parte dos professores de que conduzir os alunos a essa reflexão antecipe o início de suas vidas sexuais e crie conflito com as famílias, como foi observado na decisão dos professores de direcionar parte dos conteúdos aos alunos mais velhos, temendo desaprovação dos pais e responsáveis.

Com a necessidade de adaptação das atividades de ensino-aprendizagem para as plataformas digitais, os professores precisaram se aproximar do mundo digital, que já é familiar aos alunos, uma comunidade nativa digital. Essa proximidade aumenta a identificação entre as partes envolvidas no processo e favorece a horizontalização do ensino, tão relevantes para a discussão sobre sexualidade (ROSSI CR e FREITAS DL, 2014; OLIVEIRA RNG, et al., 2016). Esse processo foi vivenciado entre alunos e orientadores deste projeto, que também foram obrigados a migrar para o ensino digital e remoto, o que favoreceu a aproximação entre as partes, no reconhecimento dos desafios, dos ganhos e das diferenças.

Com a interrupção das atividades presenciais e a adaptação de toda rede escolar, para os meios remotos de ensino, foi necessário realizar o diagnóstico sobre o acesso dos alunos às tecnologias e à internet e se confirmou que há grande desigualdade de distribuição desse acesso e do modo como ele ocorre para os alunos da rede pública.

A escola em que foi realizado o projeto relatou que $6 \%$ dos seus alunos não tinham acesso à internet e/ou os meios para utilizá-la, o que está pouco acima do levantamento realizado pelo Comitê Gestor da Internet do Brasil em 2019, que constatou que $98 \%$ dos alunos de escolas urbanas tinham acesso à internet pelo celular (SÃO PAULO, 2019). 
Contudo, o acesso é apenas uma das variáveis no que diz respeito à utilização de tecnologias de informação por esse público e deve ser considerado junto com a forma de uso que se faz delas. Jovens de níveis socioeconômicos inferiores, como é o caso dos usuários de rede pública de ensino, utilizam a internet mais para entretenimento, como redes sociais e jogos, do que para buscar informações quando comparados a seus pares de nível socioeconômico mais elevados (CHEN W e WELLMAN B, 2007; KNOP MFP, 2017). O uso disseminado pelas classes sociais mais elevadas favorece o aproveitamento dos recursos de informação e mais habilidade na sua seleção na hora de utilizá-los como fonte de estudo, o que pode ampliar a desigualdade (CHEN W e WELLMAN B, 2007; KNOP MFP, 2017; HARRIS C, et al., 2017).

Educar é uma competência geralmente negligenciada nos cursos da área da saúde, apesar do seu papel central na construção da relação médico-paciente, cerne da prática profissional de seus graduandos (DANDAVINO M, et al., 2007; PELUSO MJ e HAFLER JP, 2011; RIBEIRO MF, et al., 2018). Neste sentido, para os alunos de medicina, esse projeto favoreceu o desenvolvimento de competências para o exercício da educação em saúde.

Todo o processo aqui relatado foi plenamente vivenciado pelos alunos envolvidos, desde o seu planejamento para a forma presencial e a articulação com as escolas, até a adaptação para os meios digitais, com o desenvolvimento do conteúdo e sua disponibilização para a escola. Isso leva não só a aquisição de conhecimento sobre sexualidade, como também sobre técnicas de ensino-aprendizagem, construção de projetos de intervenção, além de desenvolver habilidades de comunicação, gestão, resolução de problemas e trabalho em equipe, todas fundamentais para a prática médica (PEIXOTO MT, et al., 2019).

A pandemia da COVID-19 impôs o isolamento físico que foi regente das adaptações ocorridas em todo cenário de ensino no primeiro semestre de 2020. As atividades educativas realizadas através do contato com as escolas têm gerado muitas experiências positivas que se verificam a partir de novas parcerias que foram sendo construídas ao longo dos anos, permitindo aos participantes a reflexão sobre o papel e a responsabilidade da escola médica na educação em saúde, construída em colaboração com a rede de educação básica, com oportunidade de transpor barreiras e encontrar soluções criativas na adversidade.

\section{FINANCIAMENTO}

Aluna Bolsista do CNPq - Brasil e alunas Bolsistas PIBIC-CEPE - PUC/SP. Auxílio financeiro do Plano de Incentivo a Projetos de Extensão (PIPEXT) da PUC/SP, Solicitação:13856

\section{REFERÊNCIAS}

1. BRASIL. Parâmetros curriculares nacionais: terceiro e quarto ciclos de ensino fundamental. Secretaria de Educação Fundamental, Brasília, DF, 1998.

2. BRASIL. Ministério da Educação. Conselho Nacional de Educação. Resolução CNE/CES no 3, de 20 de junho de 2014. Institui Diretrizes Curriculares Nacionais do Curso de Graduação em Medicina. Brasília; 2014.

3. BRASIL. Ministério da Educação. Portaria nํ 345, de 19 de março de 2020. Altera a Portaria MEC n 343 , de 17 de março de 2020 que dispõe sobre a substituição das aulas presenciais por aulas em meios digitais enquanto durar a situação de pandemia do Novo Coronavírus - COVID-19. Diário Oficial União. Brasília, 2020.

4. CESARIO RR, et al. Alvorecer do Paradigma Sistêmico na Educação. Revista Brasileira de Educação Médica, 2019;43(1):305-313.

5. CHEN W, WELLMAN B. Minding the Cyber-gap: The Internet and Social Inequality. In: Romero M, Margolis E, editors. The Blackwell Companion to Social Inequalities. Hoboken: Blackwell Publishing, 2007. p. 523-45.

6. DANDAVINO M, et al. Why medical students should learn how to teach. Medical Teacher, 2007; 29:558-65.

7. FATMI M, et al. The effectiveness of team-based learning on learning outcomes in health professions education: BEME Guide No. 30. Medical Teacher, 2013;35(12):e1608-e1624.

8. FURLANETTO MF, et al. Educação sexual em escolas brasileiras: revisão sistemática da literatura. Cadernos de Pesquisa, 2018;48(168):550-71.

9. FURLANETTO MF, et al. Acesso e qualidade da informação recebida sobre sexo e sexualidade na perspectiva adolescente. Estudos e Pesquisa em Psicologia, 2019;19(3):644-664.

10. FURLANI J. Educação sexual: possibilidades didáticas. In: Louro GL, Neckel JF, Goellner SV, editores. Corpo, gênero e sexualidade: um debate contemporâneo na educação. Rio de Janeiro: Vozes; 2011. p. 66-81.

11. GAVA T, VILLELA WV. Educação em Sexualidade: desafios políticos e práticos para a escola. Sexualidad, Salud y Sociedad, 2016; 24:157-171. 
12. GESSER $M$, et al. Docência e concepções de sexualidade na educação básica. Psicologia \& Sociedade, 2015;27(3):558-68.

13. HARRIS C, et al. A socioeconomic related 'digital divide' exists in how, not if, young people use computers. PLoS ONE, 2017;12(3): e0175011.

14. KNOP MFP. Exclusão digital, diferenças no acesso e uso de tecnologias de informação e comunicação: questões conceituais, metodológicas e empíricas. Caderno Eletrônico de Ciências Sociais, 2017;5(2):39-58.

15. MARCON N, et al. Gravidez na adolescência: do fenômeno social a possíveis intervenções. IJDR, 2020;10(2):336905.

16. OLIVEIRA RNG, et al. Limites e possibilidades de um jogo online para a construção de conhecimento de adolescentes sobre a sexualidade. Ciência \& Saúde Coletiva, 2016;21(8):2383-92.

17. PELUSO MJ, HAFLER JP. Medical students as medical educators: opportunities for skill development in the absence of formal training programs. Yale J Biol Med, 2011;84(3):203-9.

18. PEIXOTO MT, et al. Medical education in Primary Healthcare: a multiple-approach experience to teaching, service and community integration practices. Interface (Botucatu), 2019;23(1): e170794.

19. POLES TPG, et al. Percepção dos Internos e Recém-Egressos do Curso de Medicina da PUC-SP sobre Sua Formação para Atuar na Atenção Primária à Saúde. Revista Brasileira de Educação Médica, 2019; 42(3):121-128.

20. RIBEIRO MF, et al. A avaliação de estudantes de medicina de uma faculdade de Belo Horizonte, em relação ao processo de ensino-aprendizagem da relação médico-paciente. Revista Medica de Minas Gerais, 2018;28:e-1985

21. ROSSI CR, FREITAS DL. As Tecnologias de Informação e Comunicação - TIC na formação de Professores em Educação Sexual: O caso das E-Oficinas na I COES. Educação: Teoria e Prática, 2014;24(45):96-118.

22. SANTOS ACD, et al. Relato de Experiência: Construção e Desenvolvimento do Programa de Saúde na Escola (PSE) sob a Perspectiva da Sexualidade na Adolescência. Revista Brasileira de Educação Médica, 2019; 43(4):193-9.

23. SÃO PAULO. Comitê Gestor da Internet no Brasil. TIC Educação 2019.

24. SÃO PAULO. Decreto nํ 64.864, de 16 de março de 2020. Dispõe sobre a adoção de medidas adicionais, de caráter temporário e emergencial, de prevenção de contágio pelo COVID-19 (Novo Coronavírus), e dá providências correlatas. Diário Oficial do Estado de São Paulo, 2020. 